\title{
Desarrollo de nuevas tecnologías de programación y juegos aplicados en plataformas de internet como medio didáctico de aprendizaje en línea del idioma inglés
}

Development of new programming technologies and games applied to internet platforms as a didactic means of online learning of the English language

1 Leonardo Mauricio Martínez Paredes. https://orcid.org/0000-0002-8166-5914

Escuela Superior Politécnica de Chimborazo (ESPOCH), Sede Morona Santiago,

Ecuador, lmartinezp@espoch.edu.ec

2 Nancy de las Mercedes Barreno Silva (iD https://orcid.org/0000-0001-8362-836X Escuela Superior Politécnica de Chimborazo (ESPOCH), Sede Orellana, Orellana, Ecuador. barreno@espoch.edu.ec

3 Susy Natalia Gómez Zurita Universidad Estatal Amazónica, Puyo, Ecuador. https://orcid.org/0000-0001-6762-8191 sgomez@uea.edu.ec

Cítese: $\quad$ Martínez Paredes, L. M., Barreno Silva, N. de las M., \& Gómez Zurita, S. N. (2022). Desarrollo de nuevas tecnologías de programación y juegos aplicados en plataformas de internet como medio didáctico de aprendizaje en línea del idioma inglés. ConcienciaDigital, 5(1), 208-221. https://doi.org/10.33262/concienciadigital.v5i1.2069

CONCIENCIA DIGITAL, es una Revista Multidisciplinar, Trimestral, que se publicará en soporte electrónico tiene como misión contribuir a la formación de profesionales competentes con visión humanística y crítica que sean capaces de exponer sus resultados investigativos y científicos en la misma medida que se promueva mediante su intervención cambios positivos en la sociedad. https://concienciadigital.org La revista es editada por la Editorial Ciencia Digital (Editorial de prestigio registrada en la Cámara Ecuatoriana de Libro con No de Afiliación 663) www.celibro.org.ec 


Palabras
claves:
plataforma
internet,
learning,
herramienta
didáctica,
gamificación,
inglés.

Keywords:

internet

platform, e-

learning,

didactic tool, gamification, English.
Introducción: El artículo está dedicado al problema de la creación de una plataforma de Internet para profesores de inglés; dicha plataforma de Internet es considerada por los autores como una herramienta didáctica innovadora que realiza todas las funciones de las herramientas didácticas y puede ser utilizada para el aprendizaje de inglés a distancia para un grupo de estudiantes; Objetivo: Diseñar e implementar una plataforma de internet compuesta por herramientas tecnológicas que innoven las herramientas de aprendizaje del idioma inglés con estudiantes universitarios. Metodología: Se presenta la implementación práctica de los fundamentos teóricos de las tecnologías del juego en el diseño del juego "Tour of Ecuador": se describen las metas y objetivos del juego, la mecánica y la dinámica, se dan ejemplos de tareas realizadas por los estudiantes durante el proceso del juego. Resultados: Se analizan los resultados de la aplicación del juego propuesto. Los principales métodos de enseñanza en el marco del modelo educativo sociocultural son de carácter interactivo. Las tecnologías educativas utilizadas en el proceso educativo en las diferentes etapas de la formación se distinguen por la creatividad, la improvisación, la comunicación auténtica en el idioma de destino y crean condiciones favorables para las actividades innovadoras y creativas de los estudiantes. Conclusión: Al caracterizar las plataformas de Internet disponibles, los autores llegan a la conclusión de que las opciones propuestas no satisfacen las necesidades de los profesores de lenguas extranjeras. Las tecnologías de juego, ofrece una breve descripción de las clasificaciones modernas de juegos, así como la tecnología de gamificación, sus aspectos y funciones

\section{Abstract}

Introduction: The article is devoted to the problem of creating an Internet platform for English teachers; said Internet platform is considered by the authors as an innovative didactic tool that performs all the functions of didactic tools and can be used for distance learning of English for a group of students; Objective: Design and implement an internet platform made up of technological tools that innovate the tools for learning the English language with university students. Methodology: The practical implementation of the theoretical foundations of game technologies in the design of the game "Tour of Ecuador" is presented: the goals 
and objectives of the game, the mechanics and dynamics are described, examples of tasks performed by the players are given. students during the game process. Results: The results of the application of the proposed game are analyzed. The main teaching methods within the framework of the sociocultural educational model are interactive. Educational technologies used in the educational process at different stages of training are distinguished by creativity, improvisation, authentic communication in the target language, and create favorable conditions for innovative and creative activities of students. Conclusion: By characterizing the available Internet platforms, the authors conclude that the proposed options do not meet the needs of foreign language teachers. Gaming Technologies, provides a brief description of modern game classifications, as well as gamification technology, its aspects and functions.

\section{Introducción}

En una era de rápido desarrollo de la tecnología de la información. La sociedad hace un requisito que los futuros especialistas cuenten con las destrezas y habilidades necesarias para la adquisición autónoma de conocimientos, y su aplicación en la práctica para resolver eficazmente diversos problemas, recopilar y analizar hechos, realizar generalizaciones y conclusiones razonadas, para lograr el éxito es necesario ser comunicativos, trabajar juntos en diversas situaciones, buscar una salida a situaciones conflictivas; pensar de manera crítica y creativa, encontrando formas de resolver problemas emergentes utilizando tecnologías de la información modernas; trabajar de forma independiente para mejorar su propio nivel cultural (Larenas, 2011). El desarrollo de las habilidades anteriores y las habilidades cognitivas de los estudiantes en el proceso educativo se produce debido a las tecnologías de aprendizaje activo.

En la actualidad, el principal indicador a la hora de elegir ayudas didácticas es la consecución de los niveles finales de competencia en lenguas extranjeras, desarrollado por el Consejo de Europa y que representa un sistema paneuropeo eficaz de intercambio de información. "Actividades educativas para la adquisición del idioma debe convertirse en una lección divertida y significativa y en una verdadera creatividad linguiística. Sólo en este caso, un estudiante pasará de ser un aprendiz a ser un aprendiz, adquirirá autonomía y el deseo de desarrollarse a sí mismo de acuerdo con los nuevos estándares educativos (Reyes, 2017). 
Actualmente, la informatización global ha abarcado todas las esferas de la actividad humana, incluidas la ciencia y la educación. El desarrollo de Internet y la aparición de muchos programas informáticos que simplifican el proceso de aprendizaje han cambiado significativamente el estudio de idiomas extranjeros, haciendo que sea más rápido y fácil trabajar con fuentes auténticas (de Govea, 2007).

Los programas de formación en informática tienen una serie de ventajas sobre los métodos de enseñanza tradicionales, siendo, en primer lugar, un medio de interacción interactiva audiovisual directo. Su uso en el aula junto con los métodos de enseñanza tradicionales le permite entrenar varios tipos de actividad del habla, comprender la naturaleza de los fenómenos lingüísticos, formar habilidades lingüísticas, crear situaciones comunicativas, automatizar el lenguaje y las habilidades del habla y garantizar la implementación de un enfoque individual e intensificar el trabajo independiente del alumno., y también contribuye a un aumento de la actividad cognitiva, la motivación y la calidad del conocimiento de los alumnos (Fernández, 2014).

Las tecnologías de la comunicación informática permiten implementar de una nueva forma métodos que activan la actividad creativa de los estudiantes. Pueden participar en discusiones virtuales en varios sitios educativos y foros temáticos, y realizar proyectos creativos conjuntos junto con estudiantes de diversas instituciones educativas (Alarcón, 2014).

Así, el uso de tecnologías modernas de la información y la comunicación en el proceso educativo puede considerarse como una de las formas activas de individualización del aprendizaje (Texidor, 2017).

Las propiedades inherentes de las nuevas tecnologías, como su interactividad, poli modalidad, multimedia, visualización de contenido, juegan un papel importante en el aprendizaje. Entonces, la visualización en peltre del contenido educativo, especialmente en forma lúdica e interactiva, desarrolla estilos cognitivos de pensamiento, creatividad y actividad mental de los estudiantes, y también tiene un efecto positivo en su estado psicológico y emocional (Soler, 2007).

El uso de la tecnología informática introduce una novedad heurística en el proceso de aprendizaje y crea motivación para el autoconocimiento productivo y la superación personal, y también hace que la lección sea atractiva y verdaderamente moderna, se lleva a cabo la individualización de la formación, el control y la síntesis son objetiva y oportuna (Ibáñez, 2012).

El profesor de una lengua extranjera en la escuela primaria debe contribuir a la introducción temprana de los estudiantes más jóvenes a un nuevo espacio para ellos; para formar en los niños una actitud positiva hacia su estudio posterior, debe formar 
habilidades comunicativas elementales para hablar, escuchar, leer y escribir en los escolares más pequeños, familiarizar a los escolares más pequeños con canciones extranjeras, folclore poético y de cuentos de hadas, formar algunos conceptos lingüísticos universales observados en nativos y lenguas extranjeras, desarrollando así las capacidades intelectuales, del habla y cognitivas de los estudiantes (Ortega, 2010).

En una lección de lengua extranjera moderna en la escuela primaria, se utilizan las siguientes tecnologías: orientadas a la personalidad, tecnologías de aprendizaje del desarrollo, tecnologías de juegos, tecnologías de aprendizaje por computadora, aprendizaje explicativo e ilustrativo, desarrollo creativo y, por supuesto, tecnologías que salvan la salud (Rico, 2017). Para interesar al alumno, aliviar el estrés de la lección y hacer que las clases sean emocionantes en las lecciones de lengua extranjera en la escuela primaria, se recomienda utilizar el trabajo con presentaciones y pequeños fragmentos de video al estudiar varios temas (Fuertes-Olivera, 2012).

Al graduarse de la escuela primaria, los estudiantes tienen los siguientes resultados personales, de meta-asignaturas y de asignaturas, por nombrar solo algunos de ellos. Tienen una idea del mundo multicultural, son amigables con hablantes de otro idioma, con representantes de otras culturas, interactúan con otros, determinan y formulan objetivos educativos de forma independiente, profundizan la motivación del proceso educativo, llevan a cabo un diálogo de etiqueta elemental, son capaces de hablar de sí mismos, familia, amigo, describir un objeto, una imagen, conocer la técnica de la escritura, conocer la fonética inglesa (Pérez, 2007).

Para lograr resultados en el nivel superior en las lecciones de idiomas extranjeros, se utiliza la tecnología de actividad de habla efectiva, aprendizaje reflexivo y modular, aplicando activamente la tecnología de formación de actividad cognitiva independiente, tecnologías de creatividad colectiva y tecnologías de enseñanza explicativa e ilustrativa (Posteguillo, 2002).

El uso de tecnologías de juego en las carreras de ingeniería está llamado a formar la capacidad de resolver problemas sobre la base de una elección competente de opciones alternativas: entretenidos, teatrales, comerciales, juegos de rol de imitación (Garrido, 2010).

Las tecnologías de la información y la informática implementan un sistema de aprendizaje informático basado en el diálogo "estudiante-máquina" con la ayuda de varios tipos de programas de formación (información, control).

Con el uso de tecnología interactiva, el proceso de aprendizaje se lleva a cabo en condiciones de interacción constante y activa de todos los estudiantes. El alumno y el 
profesor son sujetos iguales de aprendizaje: el aprendizaje tiene lugar en la interacción de todos los alumnos, incluido el profesor (Kazarián, 2014).

El énfasis principal en la enseñanza de idiomas extranjeros de acuerdo con el estándar educativo del Ecuador se coloca en el desarrollo de las comunicaciones, en la capacidad de una persona para comunicarse, en el dominio de un idioma en el proceso de comunicación. La capacidad del maestro juega un papel importante para interesar a los estudiantes en el próximo trabajo, para crear una situación que estimule la actividad del habla y el pensamiento, en la que el estudiante quiera hablar sobre el tema, participar en la discusión y aclarar detalles (Arellano, 2016).

El uso de juegos de rol proporciona amplias oportunidades para mejorar la actividad del habla y el pensamiento. El juego activa la imaginación y la memoria, la experiencia de vida, lo que le da al proceso de comunicación un carácter emocional. La eficacia de la enseñanza aquí se debe principalmente a una explosión de motivación, un aumento en el interés por el tema (Martín, 2013).

El juego de roles motiva la actividad del habla, ya que los estudiantes se encuentran en una situación en la que hay que decir algo, preguntar, averiguar, aclarar, probar, compartir algo con el interlocutor. Así, el foco de atención es el contenido de la conversación, que en sí mismo es un factor positivo. Los alumnos están claramente convencidos de que están aprendiendo un idioma en el que pueden comunicarse (Clark, 2004).

En el desarrollo de ciertos algoritmos para la resolución de problemas y tareas, las tecnologías de formación como sistema de actividades de desarrollo intelectual y de comunicación de los estudiantes ayudan. Finalmente, las tecnologías dialógicas son una forma de organización y un método de enseñanza basado en el pensamiento interactivo en sistemas interactivos "alumno-alumno", "profesor-autor", "alumno-autor", etc. (Clark, 2004).

\section{Metodología}

En Las preguntas emergentes se muestran las siguientes: ¿Cómo usar estas tecnologías?", "¿Qué métodos de uso de varias aplicaciones dan los resultados más rápidos?" ¿Necesidades? "," ¿Cómo actualizarlas? " En este caso, parece que muy raramente se pregunta es de suma importancia. La pregunta más importante: "¿Por qué usamos recursos digitales?"

Cabe señalar que, para que la formación sea la más eficaz, se debe revertir el orden de consideración de estos temas y empezar a planificar el uso de las tecnologías estableciendo el objetivo a alcanzar o por lograr, es decir, desde la pregunta "¿Para qué?". 
Al mirar la pregunta “¿Qué usar?”, Inevitablemente se encuentra con una gran cantidad de recursos digitales disponibles, cada uno de los cuales cuenta con un gran número de seguidores que lo prefieren como el más efectivo o revolucionario. Hay muchos recursos digitales, tanto en línea como que requieren instalación en un dispositivo que implementa los procesos de crear uno nuevo o investigar uno existente.

Estos recursos también se pueden presentar en forma de varios cursos (MOOCS, I-tunes Courses), herramientas (Google Docs, Camtasia, Explain Everything), enciclopedias y otras fuentes académicas (Google Search, Wikipedia, diccionarios independientes, Microsoft Office) y ayudar a realizar la investigación en varias etapas: recopilación de información, síntesis, supervisión adicional del proceso de estudio y trabajo con los resultados de la investigación. Así como el proceso de creación, desde la unión en grupos creativos, la planificación, y finalizando con el análisis del trabajo realizado.

La principal dificultad para elegir tecnologías digitales, planteándose en primer lugar la pregunta: "¿Qué utilizar en el proceso de aprendizaje?" y teniendo en cuenta todas estas aplicaciones es que muchas veces se desconocen las complejidades que surgen en el uso directo de los recursos seleccionados debido al desconocimiento de cómo usarlos y cómo usarlos correctamente. Es posible que el profesor no disponga de tiempo suficiente o no desee estudiar en detalle todas las posibilidades y reglas para utilizar los recursos seleccionados con antelación.

Para aprovechar al máximo nuestras aplicaciones y programas, primero debemos hacernos preguntas: “¿Por qué? ¿Cuál es el objetivo principal del uso de tecnologías informáticas en el proceso educativo?”.

Las razones pueden ser diferentes:

- $\quad$ mejorar la comprensión de lo que se está estudiando;

- aumentar el tiempo de aprendizaje alentando a los estudiantes a utilizar aplicaciones y recursos educativos fuera del aula;

- $\quad$ incrementar el nivel de eficiencia laboral del docente;

- $\quad$ desarrollo de la independencia de los estudiantes;

- aumentar el nivel de habilidades para trabajar con tecnologías informáticas;

- $\quad$ el desarrollo de cualidades de los estudiantes como la decisión y la dedicación para lograr el resultado;

- $\quad$ preparar a los estudiantes para la vida futura;

- aumentar la motivación de los estudiantes;

- $\quad$ reducción de la cantidad de recursos físicos utilizados, etc.

Por ejemplo, si el objetivo es mejorar la comprensión de los estudiantes del material o tema que se está estudiando, de deberá planear considerando aquellos recursos que 
contribuirán a la comprensión del tema. Para hacer esto, necesita mirar el problema desde diferentes ángulos. Hoy en día, Internet y varios recursos en línea ofrecen a los educadores y estudiantes un amplio acceso a la opinión de expertos sobre muchos temas. El docente no siempre puede actuar como un experto debido a que es imposible ser plenamente consciente de cada área, y también debido al hecho de que a menudo está demasiado inmerso en los problemas y necesidades de sus alumnos para evaluar objetivamente uno o más. otra pregunta. Por lo tanto, para estudiar un determinado tema, es necesario familiarizarse con la opinión de un experto que tenga una visión más completa y moderna del tema de investigación.

Para obtener la opinión de expertos, son adecuados recursos de Internet como youtube.com y ted.com con la plataforma ed.ted.com, lo que le permite crear su propia lección basada en el video propuesto. El profesor puede dividir el video en fragmentos temáticos y trabajar en la discusión de lo que vio en la lección.

Los métodos de conocimiento conjunto de las ideas y tendencias modernas y su posterior discusión, que son ampliamente utilizados por los profesores de hoy, también tienen un gran poder de motivación en la enseñanza de idiomas extranjeros. Junto con esto, la presencia de su propia computadora y dispositivos digitales con acceso a Internet para casi todos los estudiantes facilita enormemente la tarea del maestro de involucrar

La importancia de los estudiantes en el proceso de aprendizaje de una lengua extranjera a través de Internet.

Las preguntas principales cuando un docente elige tecnologías digitales son: qué usar, cómo usar y, lo más importante, por qué usar tal o cual recurso. Es necesario, en primer lugar, determinar las principales metas y objetivos de la lección en sí y, en consecuencia, el uso de las innovaciones informáticas en el marco de esta lección. Entonces, ya debemos preguntarnos qué recurso se necesita utilizar para lograr de manera más efectiva estas metas y objetivos y, finalmente, cómo funciona nuestra herramienta de capacitación elegida. Una comprensión detallada de los temas anteriores puede aumentar significativamente la participación de los estudiantes en el proceso educativo y el desarrollo de sus habilidades y habilidades necesarias para la implementación exitosa de futuras actividades profesionales.

El objetivo principal del estudio de una lengua extranjera en la escuela es la formación de la competencia comunicativa de la lengua extranjera en los escolares, es decir, la capacidad y voluntad para llevar a cabo la comunicación interpersonal e intercultural en lengua extranjera con hablantes nativos.

Para influir en los procesos de desarrollo, aprendizaje y educación de un estudiante, se necesita tecnología pedagógica. Su fórmula es bastante compleja: un conjunto de formas, 
métodos, métodos, técnicas de enseñanza y crianza especiales, utilizados sistemáticamente en el proceso educativo, a partir de las actitudes psicológicas y pedagógicas declaradas.

El tipo moderno de comunicación establece nuevas tareas para el profesor:

involucrar a cada alumno en un proceso cognitivo activo en el idioma de destino, es decir, no en el proceso de dominio pasivo del conocimiento del lenguaje, las destrezas y habilidades del habla, sino en una actividad comunicativa-cognitiva activa, de investigación y creativa;

crear condiciones para la aplicación consciente por parte de cada alumno en la práctica de los conocimientos, habilidades y habilidades adquiridos;

brindar a los escolares la oportunidad de trabajar juntos, en cooperación en la resolución de diversos problemas, así como el libre acceso a la información necesaria para utilizarla en sus propias declaraciones.

El método de enseñar a hablar, se alejó de la memorización tradicional de los mismos temas, escribiendo un diccionario al texto y llegó a la tecnología de crear campos léxicos y semánticos sobre temas requeridos para el dominio de la le gua extranjero, moviendo a la acumulación de vocabulario de diferentes fuentes. En determinadas etapas de la formación, se prepara un enunciado nomológico oral de acuerdo con el plan propuesto utilizando campos léxicos y semánticos.

Uso Las tecnologías de la enseñanza explicativa e ilustrativa son la versatilidad y la abundancia de información, el uso rico de la visualización y las ayudas técnicas para la enseñanza que proporcionan una asimilación rápida y duradera de la información y el dominio de las formas de acciones prácticas.

\section{Resultados}

El contenido semántico de valores del concepto de "educación en lenguas extranjeras" prevé el dominio de los estudiantes no solo por la lengua extranjera en sí, sino también por la familiarización con la literatura, la historia y, en general, con la cultura del país de destino. idioma, por lo tanto, el proceso de enseñanza de idiomas extranjeros en el nivel superior plantea tareas aún más difíciles. Al expandir el círculo de culturas a través de la entrada de los estudiantes en el espacio sociocultural, al comunicarse en el FL, se forma su orientación de valores y la cultura de la personalidad misma, su desarrollo intelectual.

Una lengua extranjera en la sociedad moderna es cada vez más demandada no solo como medio de comunicación, sino también como instrumento de actividad cognitiva y profesional. En este sentido, cobra relevancia abordar la problemática de la educación bilingüe, que actúa como una forma alternativa de aprendizaje de una lengua extranjera, 
la cual, a su vez, se transforma de la meta de aprendizaje en un medio de comprensión del mundo de los saberes especiales y educación multicultural.

Los maestros que trabajan en la escuela secundaria se enfrentan a la tarea de formar competencias socioculturales y estereotipos de comportamiento, desarrollar la independencia, la capacidad de educarse a sí mismos, trabajar con programas multimedia, diccionarios electrónicos y recursos en idiomas extranjeros de Internet.

Fomentar la tolerancia y el respeto por los valores espirituales de diferentes países y pueblos, ampliar el vocabulario y, por supuesto, prepararse para el Examen de suficiencia en inglés.

Además de las tecnologías anteriores, las más populares en el nivel superior son la tecnología de la actividad del habla efectiva, la tecnología del aprendizaje reflexivo, las tecnologías para la formación de la actividad cognitiva independiente, las tecnologías de la información de la enseñanza, la tecnología del pensamiento crítico y las tecnologías de proyectos.

Tecnología "Pensamiento crítico y análisis de la información proporcionada tiene tres etapas de organización del proceso educativo.

1. Despertando interés por el tema. Es necesario actualizar los conocimientos y significados de los estudiantes en relación con el material que se está estudiando, para despertar un interés cognitivo en el material estudiado, para ayudar a los estudiantes a determinar la dirección en el estudio del tema por sí mismos.

2. Comprensión del material mientras se trabaja en él. Es necesario ayudar a percibir activamente el material que se está estudiando, para ayudar a relacionar el conocimiento antiguo con el nuevo.

3. Generalización del material, resumiendo. El profesor debe ayudar a los estudiantes a generalizar de forma independiente el material que se está estudiando, ayudar a determinar de forma independiente las direcciones en el estudio posterior del material.

Al trabajar con estudiantes de secundaria, es efectivo utilizar tecnologías estructurales y lógicas que representan una organización por fases del sistema de formación, proporcionando una secuencia lógica para la formulación y solución de tareas didácticas basadas en una elección adecuada de contenido, formas, métodos y ayudas didácticas en cada etapa, teniendo en cuenta el diagnóstico por fases de los resultados ... Las tecnologías de integración son ampliamente utilizadas en el aula como sistemas didácticos que aseguran la integración de diversos conocimientos y habilidades, diversos tipos de 
actividades a nivel de cursos integrados, temas educativos, problemas, lecciones de otras formas de organización de la formación.

El uso del método del proyecto brinda un aumento significativo en el conocimiento y las habilidades de los estudiantes, contribuye a la acumulación de su experiencia de vida y sabiduría.

Trabajar en un proyecto genera tareas, ideas, solución e implementación que, una persona, de manera independiente o con la ayuda de otras personas, se orienta en la realidad, adquiere conocimientos sobre el mundo y las formas de comportarse en él, y, por tanto, se forma.

El proyecto es una forma tan abierta y dinámica de organizar las actividades educativas de los estudiantes y las actividades pedagógicas del docente, lo que implica su elección y soluciones creativas que conduzcan al desarrollo de los estudiantes. La popularidad de esta tecnología y sus perspectivas se explica, en primer lugar, por el hecho de que el proyecto conecta directamente el proceso de dominar un determinado conocimiento de la materia con el uso real de este conocimiento.

El conocimiento deja de ser un objetivo, pero se convierte en un medio en la educación genuina, que ayuda a dominar los patrones de pensamiento, a formar las propias estrategias de pensamiento, lo que permite a todos dominar de forma independiente la acumulación de conocimientos.

La cognición exitosa del mundo de las lenguas extranjeras y la comprensión de la lengua materna de uno solo es posible bajo la condición de una actividad cognitiva intensiva de los estudiantes, su creatividad intelectual activa, así como en el curso de la aplicación de la investigación independiente.

\section{Conclusiones}

- El uso de los recursos de Internet en la enseñanza de lenguas extranjeras permite crear las condiciones para el desarrollo de todo lo necesario y correspondiente a las realidades de modernidad de las competencias de los estudiantes.

- Para el desarrollo de las habilidades investigativas, de acuerdo con el Estándar Educativo Superior, el docente debe ampliar los horizontes y mejorar la cultura de la información de los estudiantes

- Trabajar en un proyecto ayuda a los alumnos a comprender el papel del conocimiento en la vida y el aprendizaje.

- En la educación moderna, un proyecto se define como pensar en el futuro, sugerir, diseñar, proyectos que son propios de cada persona en cualquier etapa de su desarrollo. 
- El profesor debe ayudar a los estudiantes a generalizar de forma independiente el material que se está estudiando, ayudar a determinar de forma independiente las direcciones en el estudio posterior del material.

\section{Referencias bibliográficas}

Alarcón, D. N. (2014). Las Tecnologías de la Información y Comunicación (TIC) y su relación con el aprendizaje del idioma inglés en los estudiantes de la especialidad de inglés-francés, promoción 2011 de la Uni.

Arellano, M. D. (2016). La educación informal en el aprendizaje del inglés como lengua extranjera. Tejuelo: Didáctica de la Lengua y la Literatura. Educación, (24), 6891.

Clark, A. S. (2004). Diccionario Inglés a Español de Computación e Internet: English-toSpanish Computer and Internet Dictionary. Universal-Publishers.

de Govea, A. F. (2007). Principios Cognitivo-constructivistas en la Aplicación de las Tecnologías de la Información y la Comunicación (TIC) para la EnseñanzaAprendizaje de Inglés con Propósitos Específicos. Principios, 3, 38-64.

Fernández, N. M. (2014). Los estudiantes adultos mayores y las nuevas tecnologías en el aprendizaje de inglés en la UNLaM. Recuperado de: https://www. oei. es/histórico/congreso2014/memoriactei/510. pdf.

Fuertes-Olivera, P. A. (2012). La lexicografía de Internet: el" Diccionario inglés-español de contabilidad". CÍRCULO de Lingüística Aplicada a la Comunicación, 52, 21.

Garrido, J. (2010). Lengua y globalización: inglés global y español pluricéntrico. Historia y comunicación social, 15, 63-95.

Ibáñez, N. (2012). Resignificado del aprendizaje del idioma inglés bajo el influjo de las tecnologías de la información y comunicación. Caso: Universidad de Carabobo. Orbis. Revista Científica Ciencias Humanas, 8(22), 49-63.

Kazarián, Y. (2014). Actividades para motivar el aprendizaje de los estudiantes en las clases de inglés. Revista Habanera de Ciencias Médicas, 13(4), 612-622.

Larenas, C. H. (2011). El aprendizaje del inglés y el uso de tecnologías: percepciones de estudiantes y profesores de inglés del nivel secundario chileno. Matices en Lenguas Extranjeras, (5), 1-37.

Martín, D. (2013). Inglés, Internet y Pizarra Digital. Daniel Martin. 
Ortega Martín, J. L. (2010). Efecto de las nuevas tecnologías en el aprendizaje del inglés en primaria: un currículo compartido por colegios de Francia, Inglaterra y España.

Pérez Sabater, C. (2007). Los elementos conversacionales en la comunicación escrita vía Internet en lengua ingles. Universitat Jaume I.

Posteguillo, S. (2002). La influencia del inglés de Internet en la lengua española. Revista de investigación lingüística, Vol. 5, ․․ 2 (2002).

Reyes, N. E. (2017). Propuesta metodológica para el aprendizaje de inglés en la Universidad Tecnológica Equinoccial con el uso de las TIC. (Doctoral dissertation, Universidad de Extremadura).

Rico Sirvent, C. (2017). La ayuda de las Nuevas Tecnologías en el aprendizaje del inglés como lengua extranjera.

Soler Costa, R. (2007). Nuevo enfoque metodológico a través de las TIC en el proceso de enseñanza-aprendizaje del inglés: estrategias de aprendizaje en el entorno virtual. Revista interuniversitaria de formación del profesorado.

Texidor Pellón, R. R. (2017). Las tecnologías de la información y la comunicación en la enseñanza de inglés en Ciencias Médicas. Educación Médica Superior, 31(2), 00 .

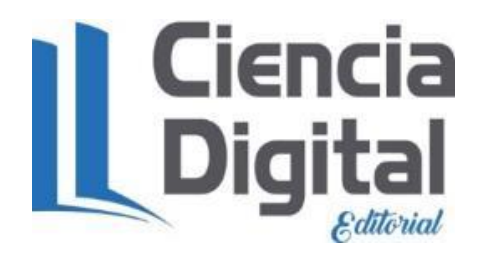


El artículo que se publica es de exclusiva responsabilidad de los autores y no necesariamente reflejan el pensamiento de la Revista Conciencia Digital.

\section{Ciencia}

El artículo queda en propiedad de la revista y, por tanto, su publicación parcial y/o total en otro medio tiene que ser autorizado por el director de la Revista Conciencia Digital.
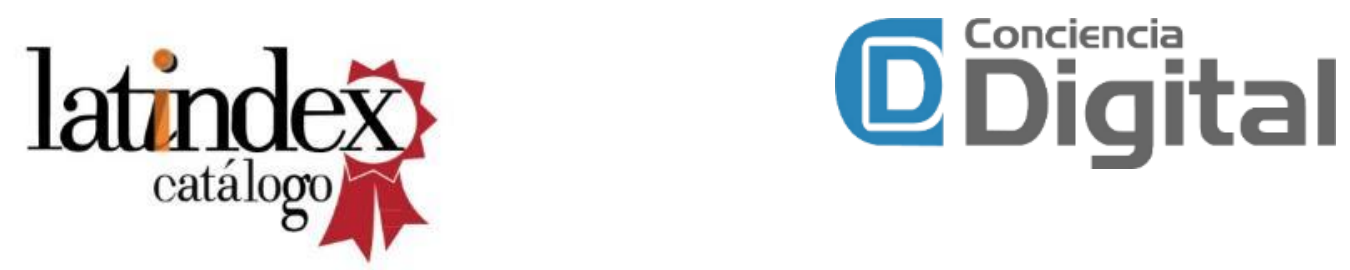

Indexaciones

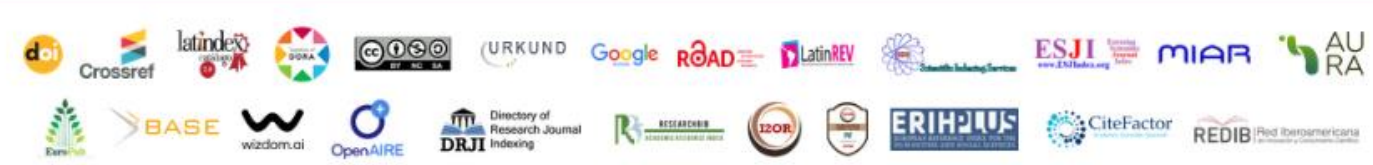

